\section{HEWLETT-PACKARD EUROPHYSICS PRIZE}

\section{Coherent Phenomena in Disordered Conductors}

The 1993 Hewlett-Packard Europhysics Prize of EPS for outstanding achievements in condensed matter physics has been awarded for "pioneering theoretical work on coherent phenomena in disordered conductors" to:

Boris L. Altshuler (Massachusetts Institute of Technology, Cambridge, USA) Arkadii G. Aronov, David E. Khmelnitskii and Anatoly L. Larkin (A.F. Ioffe Physico-Technical Institute, St. Petersburg)

Boris Z. Spivak (University of Washington, Seattle, USA).
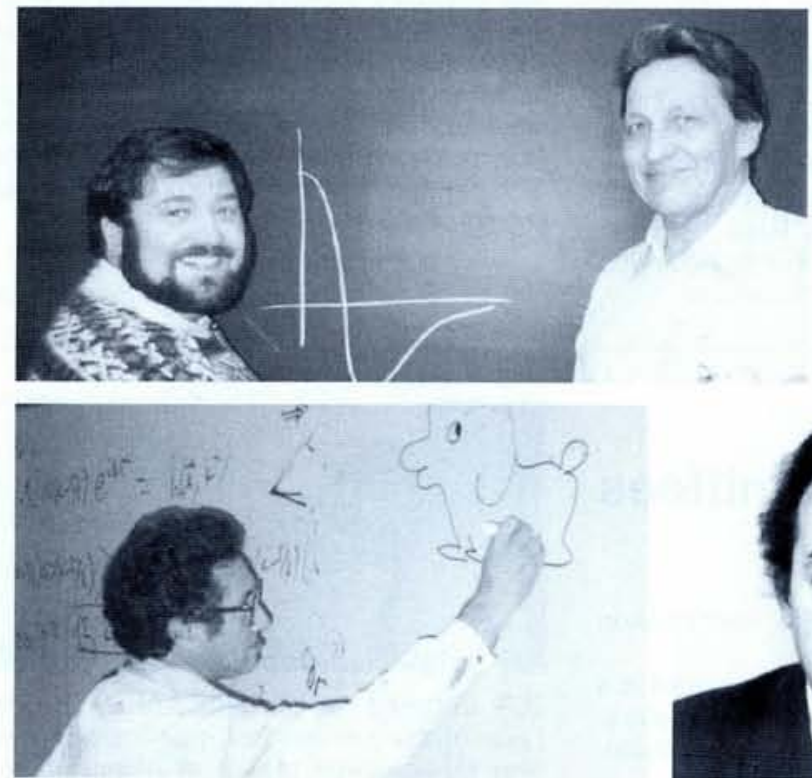

Clockwise from the upper left:

A.L. Larkin and B.L. Altshuler (on the right); B.Z. Spivak; A.G. Aronov; D.E. Khmelnitskii.

B.L. Altshuler has been Professor of Physics at the Massachusetts Institute of Technology since 1990. He was born in St. Petersburg in 1955, studied at the St. Petersburg State University, and received his Ph.D. in 1979 from the St. Petersburg Institute for Nuclear Physics where he then worked as a Research Fellow until 1989.

A.G. Aronov, Department Head at the A.F. loffe Physico-Technical Institute, St. Petersburg, since 1990, is presently a Visiting Professor at the Weizmann Institute, Rehovot, Israel. Born in 1939 in St. Petersburg, where he graduated from the Institute of Electrotechnical Engineering and was awarded a Ph.D. from the Institute of Semiconductors. His Habilitation in 1977 is from the St. Petersburg Institute for Nuclear Physics where he worked in 1974-90 after spending two years at the loffe Institute. He spent a year as a Humbolt Fellow at the Institute for the Theory of Condensed Matter, University of Karlsruhe, Germany, in 1991-92 and has been a visiting scientist in various other institutes in Germany, the USA, and Japan. Professor Aronov was a Corresponding Member of the former USSR Academy of Sciences.

D.E. Khmelnitskii has been a Senior Research Fellow at Trinity College Cambridge, UK, since 1991 and is attached to the Cavendish Laboratory, Cambridge. He remains on the staff of the L.D. Landau Institute of Theoretical Physics, Moscow, where he has worked since 1971 apart from four years spent importance of the work are also very helpful. sics Prize, EPS, P.O. Box 69, CH-1213 Petit-Lancy 2.
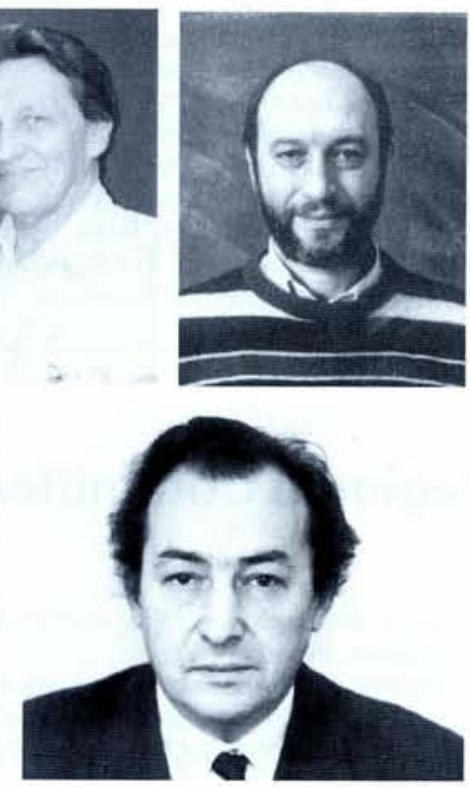

The prizewinners have profoundly influenced our understanding of the physics of transport phenomena in disordered metals and semiconductors. By introducing new notions, they were able to predict and explain numerous very interesting and subtle effects.

In particular, it was shown that in certain cases, the full quantum mechanical treatment of conduction electrons is indispensable, and can lead to essential quantum corrections to the classical description of electrical conductivity. This analysis is based on the concept of quantum mechanical trajectories, where the phase of the amplitude is conserved after multiple elastic scattering of electrons on defects in impure conductors; the phase conservation leads to interference effects between the amplitudes for different paths of the electrons. These effects are particularly pronounced for electrons returning to the points from whence they started (e.g., in closed loops) where interference causes an apparent enhancement of the interaction between the electrons, resulting in quantum corrections to the classical description of electrical conductivity. Inelastic scattering destroys the phase coherence, and the interference is lost.

Following another line of reasoning, involving once again the effect of disorder and the concept of interference, the prizewinners

1932, he graduated with a Ph.D. from the I.V. Kurchatov Institute of Atomic Energy, Moscow, in 1965. A Corresponding Member of the former USSR Academy of Sciences, he is now a member of the Russian Academy of Sciences. Professor Larkin is presently visiting the A.T.\&T. Bell Laboratories, Murray Hill, NJ, USA, having spent six months at Chicago University; he will shortly visit the Weizmann Institute, Rehovot.

with the Institute for Solid-State Physics, Chernagolovka. Dr. Khmelnitskii was born in 1944 in Moscow and graduated in 1969 from the Moscow Institute of Physical Engineering. He was awarded his Ph.D. and his Habilitation from the Landau Institute.

A.L. Larkin has been a Department Head at the Landau Institute from 1966 and Professor of Theoretical Physics at Moscow State University since 1967. Born in Moscow in
B.Z. Spivak has been Professor of Physics at the University of Washington, Seattle, WA, USA, since 1991 after spending a year as a Visiting Professor at the University of California, Los Angeles, CA. He was educated at the St. Petersburg Polytechnic Institute, receiving his Ph.D. in 1987. He worked on the research staff of the Sciences and Technology Corporation of the former USSR Academy of Sciences in 1986-89.

\title{
CALL FOR NOMINATIONS
}

\section{Hewlett-Packard Europhysics Prize}

The Selection Committee for the Hewlett-Packard Europhysics Prize invites nominations for the 1994 award. The Prize is give for an outstanding contribution to condensed matter physics within the previous five years, with the potential for leading advances in electronic, electrical or materials engineering. Nominations may be submitted by EPS members as individuals or as representatives of a Division, Section or Group.

Previous winners have been H. Ibach, G. Ertl and J.P. Toennies (1992) and D. Jérome and K. Bechgaard (1991). To maintain this extremely high standard, it is necessary that the Committee receive proposals which represent the breadth and strength of European condensed matter physics. Proposals should indicate the motivation for the award and include, if possible, a brief curriculum vitae of each of the nominees and a short list of major publications. Letters of support from authorities in the field which outline the

All information will be treated as strictly confidential, and although proposals will be acknowledged, there will be no further correspondence. EPS members are urged to submit a nomination before 30 June 1993 to the Selection Committee, H.-P. Europhy-

P. Wyder, Chairman, Selection Committee 
were able to take into account Coulomb repulsion between electrons, leading to corrections for electron-electron scattering. This led to a theory for the negative value of the coefficient of magnetoresistance in disordered metals - a phenomenon which has puzzled physicists for more that 30 years. The same reasoning also gave a long-sought explanation for the zero-voltage-anomaly in tunnel junctions, and predicted the unusual temperature and magnetic field dependencies of resistivity and the Hall effect in twodimensional electron gases and in thin metallic films.

In addition, it was demonstrated that interference phenomena are particularly interesting if magnetic fields are present. Owing to the Bohm-Aharanov effect, the magnetic flux through a closed loop introduces changes in the interference pattern which are periodic in the magnetic field. This leads to the famous and remarkable prediction of the oscillatory behaviour of the resistance of thin hollow cylinders of a normal metal in a field parallel to the axis of the cylinder; it was confirmed beautifully by the spectacular SharvinSharvin experiment (see figure). This work, in effect, opened up in a very fundamental way a new branch of physics which we now call mesoscopics.

The complete calculations of quantum corrections to electrical conductivity and other

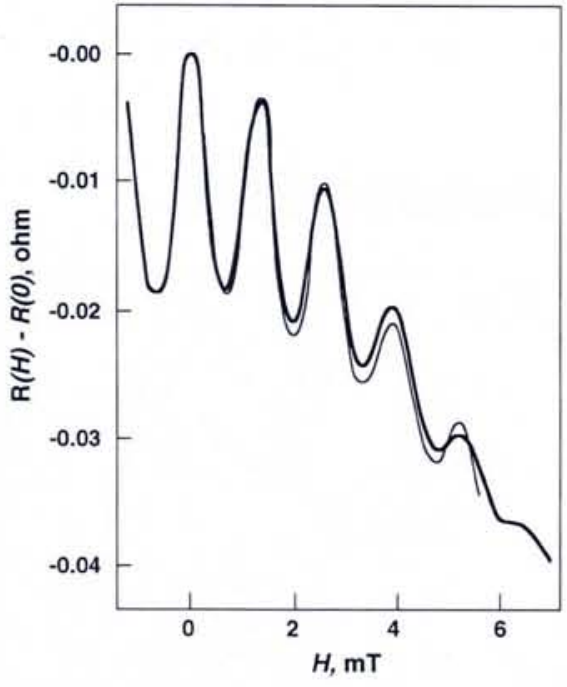

physical characteristics of disordered metals are usually performed using the powerful methods of quantum fieid theory, as pioneered by the Russian school. But the prizewinners also introduced very simple and very elegant reasoning which make it possible to obtain order-of-magnitude estimates of the corrections, and to give a very clear physical picture of the origin of the various effects. The winners belong directly or indirectly to
The Sharvin-Sharvin experiment [Altshuler B.L. et al., JETP Lett. 35 (1982) 5881]: owing to the dependence of electron wave functions on the vector potential of a magnetic field (the Bohm-Aharanov effect), the conductivity of a long, thin-walled cylinder made of a normal metal is an oscillatory function of the magnetic flux which passes through the cylinder. The curve (thin line) calculated using the theory of B.L. Altshuler, A.G. Aronov and B.Z. Spivak [JETP Lett. 33 (1981) 94] for the magnetoresistance at liquid helium temperatures of a Li cylinder with length $10 \mathrm{~mm}$, diameter $1.1 \mu \mathrm{m}$, and wall thickness $0.12 \mu \mathrm{m}$ fits the experimetal curve (thick line) using only these parameters.

the great and famous Landau school of theoretical physics for which a clear physical understanding and interpretation is as important as exact formal results. Working in this spirit and with this approach, the prizewinners have togther made far reaching and stimulating discoveries while based in two different places, in Moscow and in St. Petersburg. It may well be possible that their work will eventually lead to novel and important types of electronic devices and sensors.

P. Wyder, Grenoble

\section{FSU SMALL GRANTS PROGRAMME}

\section{Supplementary Grants for EC\&E}

The American Physical Society (APS) decided last month to extend its small grants programme for direct aid to physicists in the former Soviet Union to include grants to east and central Europe (including the former Yugoslavia if conditions permit, and excluding Germany). The maximum grant is \$US 1000.- per individual and the funds must be used to supplement existing funds for FSU physicists to enable an existing collaboration with a group in E\&CE to be maintained after the physicists return home to the FSU.
To apply, group leaders in internationally recognized laboratories in E\&CE should submit by 15 April 1993 a proposal on behalf of FSU physicists to Irving Lerch, Director, International Affairs, APS, 335 East 45th St. New York, NY 10017, USA [tel./fax $+1-212$ 6827341 / 68725 32; apsap @ cunyvm]

The proposal should describe the collaboration, the facilities available to the FSU physicists, details of the physicists, and how the grant would be spent. A laboratory can apply for grants for up to four individuals.

\section{Programme Restructures}

The APS Small Grants Programme [see EN 23 (1992) 139] was set up in 1992 by an ad hoc APS task force on the crisis of basic research in the FSU. It aimed to provide SUS 500.- short-term survival mode grants directly to FSU physicists unable to travel or work abroad. Applications were channelled through a Moscow Coordination Committee (MCC) appointed by the task force. About \$US 900000.- has been distributed so far, mainly as small grants, but fellowships are also authorised. The MCC recommended in December that the programme be extended for a second year.

The MCC started last September to set up advisory committees based in the FSU republics to help it in its work; applications are processed by the committees before being forwarded to the task force. The MCC continues to certify that a laboratory has received funds so that matching funds provided, for example, by the Russian research minister can be sought (the Minister has also exempted grants from tax)

The MCC intends to enlarge its scope by becoming the Russian International Coordinating Committee on International Collaboration in Basic Science which will aim to obtain bilateral support for international research partnerships. The task force has been dissolved in the meantime, and grants are now approved by Bill Blanpied, a member (and immediate past-Chairman) of the APS Committee on International Scientific Affairs (CISA), who calls upon a network of advisors.

The task force's work has up to now been carried out by CISA operating from the APS secretariat. But with the increase in the volume of work and the need to move towards longer term and more stable forms of support, administration of the small grants programme will be taken over in mid- 1993 by the

\section{George Soros \\ Benefactor Extraordinaire}

George Soros grew up in Hungary and moved to London in 1947 to study at the London School of Economics. He worked as an investment manager, first in London and later in New York where he established the Quantum Fund - an investment fund reported to be now worth 7000 MSUS. The Open Society Fund, his first foundation, was set up in 1979 to support students and human rights activists in South Africa. It was followed in 1984 by a fund for his native Hungary that developed into a network of foundations for east and central Europe, some of which bear his name. Spin-offs include the Central European University, that gives graduate courses in Budapest and until next year in Prague, and the EastWest Management Institute that places managers for a short time in western companies.

International Science Foundation (ISA). This private body was established recently by George Soros (see box), the Hungarian financier, who made a widely reported commitment last December to donate 100 MSUS. ISA aims to support scientific culture in the FSU by offering grants, fellowships and endowments to both both individuals and institutions. E.W.A. Lingeman, Secretary of the EPS East-West Coordination Committee, who attended a CISA meeting in New York in January reports that ISA will soon launch an approximately 6 MSUS programme involving emergency six-month support for small research groups; a much larger Phase II programme should start in July 1993. An Executive Director and the Foundation's Board have not yet been formally appointed. 